\title{
Total domination versus paired domination
}

\author{
Oliver Schaudt \\ Institut für Informatik \\ Universität zu Köln \\ Weyertal 80, 50931 Cologne, Germany \\ schaudt@zpr . uni-koeln.de
}

April 12, 2011

\begin{abstract}
A dominating set of a graph $G$ is a vertex subset that any vertex of $G$ either belongs to or is adjacent to. A total dominating set is a dominating set whose induced subgraph does not contain isolated vertices. The minimal size of a total dominating set, the total domination number, is denoted by $\gamma_{t}$. The maximal size of an inclusionwise minimal total dominating set, the upper total domination number, is denoted by $\Gamma_{t}$. A paired dominating set is a dominating set whose induced subgraph has a perfect matching. The minimal size of a paired dominating set, the paired domination number, is denoted by $\gamma_{p}$. The maximal size of an inclusionwise minimal paired dominating set, the upper paired domination number, is denoted by $\Gamma_{p}$.

In this paper we prove several results on the ratio of these four parameters: For each $r \geq 2$ we prove the sharp bound $\gamma_{p} / \gamma_{t} \leq 2-2 / r$ for $K_{1, r}$-free graphs. As a consequence, we obtain the sharp bound $\gamma_{p} / \gamma_{t} \leq 2-2 /(\Delta+1)$, where $\Delta$ is the maximum degree. We also show for each $r \geq 2$ that $\left\{C_{5}, T_{r}\right\}$-free graphs fulfill the sharp bound $\gamma_{p} / \gamma_{t} \leq 2-2 / r$, where $T_{r}$ is obtained from $K_{1, r}$ by subdividing each edge exactly once. We show that all of these bounds also hold for the ratio $\Gamma_{p} / \Gamma_{t}$. Further, we prove that a graph hereditarily has an induced paired dominating set iff $\gamma_{p} \leq \Gamma_{t}$ holds for any induced subgraph. We also give a finite forbidden subgraph characterization for this condition. We exactly determine the maximal value of the ratio $\gamma_{p} / \Gamma_{t}$ taken over the induced subgraphs of a graph. As a consequence, we prove for each $r \geq 3$ the sharp bound $\gamma_{p} / \Gamma_{t} \leq 2-2 / r$ for graphs that do not contain the corona of $K_{1, r}$ as subgraph. In particular, we obtain the sharp bound $\gamma_{p} / \Gamma_{t} \leq 2-2 / \Delta$.

keywords: total domination, upper total domination, paired domination, upper paired domination, generalized claw-free graphs.
\end{abstract}

MSC: $05 \mathrm{C} 69$

\section{Introduction}

In this paper we consider finite, simple and undirected graphs. Since this paper deals with graph invariants, we are allowed to treat isomorphic graphs as identical. All considered graphs are assumed to be free of isolated vertices. This assumption is necessary and can be made without loss of generality, since total 
dominating sets and paired dominating sets do not exist in graphs that have isolated vertices.

Let $G$ be a graph. If a graph $H$ is an induced subgraph of $G$, then we write $H \sqsubseteq G$. If $H \nsubseteq G$, then $G$ is said to be $H$-free. If $\mathcal{H}$ is a set of graphs, then $G$ is said to be $\mathcal{H}$-free if $G$ is $H$-free for every $H \in \mathcal{H}$. The corona of $G$, denoted by $\operatorname{Cr}(G)$, is the graph obtained from $G$ by attaching a pendant vertex to each vertex of $G$. The complete bipartite graph $K_{1,3}$ is called the claw. In general, graphs of the form $K_{1, r}$ are sometimes called generalized claws. The path on three vertices we denote by $P_{3}$. Note that $P_{3} \cong K_{1,2}$. For each $r \geq 3$, the graph $T_{r}$ is obtained from $K_{1, r}$ by subdividing each edge exactly once. The claw, $T_{3}$ and the corona of the claw are displayed in Figure 1.
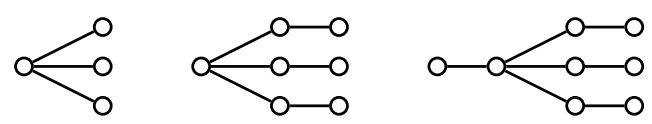

Figure 1: $K_{1,3}, T_{3}$ and $\operatorname{Cr}\left(K_{1,3}\right)$.

A dominating set $X$ of $G$ is a vertex subset such that any vertex of $V(G) \backslash X$ has a neighbor in $X$. There is a lot of literature dealing with the concept of dominating sets and its many variants. An introduction into the field of domination in graphs is the book by Haynes, Hedetniemi and Slater [1]. A total dominating set is a dominating set $X$ whose induced subgraph, denoted by $G[X]$, does not have isolated vertices. Since our considered graphs do not have isolated vertices, any graph has a total dominating set. The minimal size of a total dominating set, the total domination number, is denoted by $\gamma_{t}$. An inclusionwise minimal total dominating set is said to be minimal. The maximal size of a minimal total dominating set, the upper total domination number, is denoted by $\Gamma_{t}$. Total domination was introduced by Cockayne, Dawes and Hedetniemi [2] in 1980 and is well studied now. A survey on total domination, including many recent results, is the paper by Henning [3]. A paired dominating set is a dominating set whose induced subgraph has a perfect matching. Again, since our considered graphs do not have isolated vertices, any graph has a paired dominating set. The minimal size of a paired dominating set, the paired domination number, is denoted by $\gamma_{p}$. An inclusionwise minimal paired dominating set is said to be minimal. The maximal size of a minimal paired dominating set, the upper paired domination number, is denoted by $\Gamma_{p}$. Paired domination was introduced by Haynes and Slater [4] in 1998 and has received much attention in the literature. There are a lot of papers dealing with this topic, some of which are stated below. An induced paired dominating set is a paired dominating set that induces a 1-regular subgraph. So to say, it is an induced matching whose matched vertices dominate the graph. Not every graph has an induced paired dominating set, e.g. the graphs in Figure 2. In fact, it is an $\mathcal{N} \mathcal{P}$-complete problem to decide if a given graph has such a set, as is shown by Telle [5]. Induced paired dominating sets are also studied by Haynes, Lawson and Studer [6] and Zelinka [7].

Especially for claw-free graphs, a lot of research has been done on total domination $[8,9,10,11]$ and paired domination $[12,13,14]$. Besides having bounds on the parameters themselves, it is also interesting to know how the parameters behave compared to each other. Usually, the absolute difference of two parameters is not bounded. In fact, $\gamma_{p}-\gamma_{t}, \Gamma_{p}-\Gamma_{t}$ and $\gamma_{p}-\Gamma_{t}$ can grow 
arbitrarily large, as can be easily seen considering the graphs $C r\left(K_{1, r}\right)$. In this case, it is more promising to study the ratios of the parameters, i.e. $\gamma_{p} / \gamma_{t}$, $\Gamma_{p} / \Gamma_{t}$ and $\gamma_{p} / \Gamma_{t}$.

In [4], the following bound was given:

Theorem 1 (Haynes and Slater [4]). Let $G$ be a graph. Then

$$
\gamma_{p}(G) \leq 2 \gamma_{t}(G)-2 .
$$

Hence,

$$
\frac{\gamma_{p}}{\gamma_{t}} \leq 2
$$

holds in general. Since $\gamma_{p}\left(T_{r}\right) / \gamma_{t}\left(T_{r}\right)=2-2 /(r+1)$ for each $r \geq 1$, (1) is asymptotically sharp. The same holds for the ratio $\Gamma_{p} / \Gamma_{t}$, as is shown by Dorbec, Henning and McCoy [16].

However, if certain subgraphs are forbidden, (1) can be improved. For example, Brigham and Dutton [15] show that for claw-free graphs it holds that $\gamma_{p} / \gamma_{t} \leq 4 / 3$. One of the aims of this paper is to generalize this bound to generalized claw-free graphs. Further, we establish similar bounds for the ratio $\gamma_{p} / \Gamma_{t}$.

\section{The main results}

In this section we present our main results. The proofs are given in section 3.

We improve (1) by proving the following two theorems. The first one is a bound in terms of the smallest generalized claw that is not contained in the considered graph:

Theorem 2. Let $G$ be a $K_{1, r}$-free graph for some $r \geq 3$. Then

$$
\frac{\gamma_{p}(G)}{\gamma_{t}(G)} \leq 2-\frac{2}{r}
$$

and this bound is sharp for each $r \geq 3$. Further,

$$
\frac{\Gamma_{p}(G)}{\Gamma_{t}(G)} \leq 2-\frac{2}{r} .
$$

As a consequence, we obtain

Corollary 1. If $G$ is a graph with maximum degree $\Delta$,

$$
\frac{\gamma_{p}(G)}{\gamma_{t}(G)} \leq 2-\frac{2}{\Delta+1}
$$

and this bound is sharp for each $\Delta$. Further,

$$
\frac{\Gamma_{p}(G)}{\Gamma_{t}(G)} \leq 2-\frac{2}{\Delta+1} .
$$

Note that (3) and (5) are possibly not sharp.

The second theorem is a strengthening of Theorem 2, but is only applicable to $C_{5}$-free graphs: 
Theorem 3. Let $G$ be a $\left\{C_{5}, T_{r}\right\}$-free graph for some $r \geq 3$. Then

$$
\frac{\gamma_{p}(G)}{\gamma_{t}(G)} \leq 2-\frac{2}{r}
$$

and

$$
\frac{\Gamma_{p}(G)}{\Gamma_{t}(G)} \leq 2-\frac{2}{r}
$$

Both bounds are sharp for each $r \geq 3$.

The next Theorem deals with the interplay of the ratio $\gamma_{p} \leq \Gamma_{t}$ and the existence of induced paired dominating sets: If a graph $G$ has an induced paired dominating set $P$, then $P$ is also a minimal total dominating set. Hence, $|P| \leq \Gamma_{t}(G)$. In particular, $\gamma_{p}(G) / \Gamma_{t}(G) \leq 1$. Theorem 4 gives the opposite direction: If $\gamma_{p}(H) / \Gamma_{t}(H) \leq 1$ holds for any induced subgraph of $G$, then $G$ also has an induced paired dominating set. Furthermore, it gives the forbidden subgraph characterization for this condition. According to our knowledge, this characterization has not been discovered yet.

Theorem 4. Let $G$ be a graph. The following statements are equivalent:

1. Any induced subgraph $H$ of $G$ has an induced paired dominating set.

2. $\max _{H \sqsubseteq G} \gamma_{p}(H) / \Gamma_{t}(H)=1$.

3. $G$ is $\left\{C_{5}, \operatorname{Cr}\left(K_{3}\right), C r\left(P_{3}\right)\right\}$-free.

The forbidden subgraphs of Theorem 4 are displayed in Figure 2.


Figure 2: $C_{5}, \operatorname{Cr}\left(K_{3}\right)$ and $\operatorname{Cr}\left(P_{3}\right)$.

Theorem 5 deals with the ratio $\gamma_{p} / \Gamma_{t}$. It completely determines the maximal value of the ratio $\gamma_{p} / \Gamma_{t}$ taken over the induced subgraphs of a graph. Furthermore, it provides a complete list of the possible values and gives a finite forbidden subgraph characterization for each value.

Theorem 5. Let $G$ be a graph and let

$$
\lambda=\max \left\{2, \min \left\{r: G \text { is } C r\left(K_{1, r}\right)-\text { free }\right\}\right. \text {. }
$$

Then

$$
\max _{H \sqsubseteq G} \frac{\gamma_{p}(H)}{\Gamma_{t}(H)}=\left\{\begin{array}{ll}
2-\frac{2}{\lambda}, & \text { if } G \text { is }\left\{C_{5}, C r\left(K_{3}\right)\right\} \text {-free } \\
\max \left\{\frac{4}{3}, 2-\frac{2}{\lambda}\right\}, & \text { otherwise }
\end{array} .\right.
$$

The possible values of $\max _{H \sqsubseteq G} \gamma_{p}(H) / \Gamma_{t}(H)$ provided by Theorem 5 are displayed in Table 1.

As a direct consequence of Theorem 5, we obtain the following bound: 


\begin{tabular}{c|c}
\hline Property of a graph $\boldsymbol{G}$ & $\boldsymbol{m a x}_{\boldsymbol{H} \sqsubseteq \boldsymbol{G}} \gamma_{\boldsymbol{p}}(\boldsymbol{H}) / \boldsymbol{\Gamma}_{\boldsymbol{t}}(\boldsymbol{H} \boldsymbol{)}$ \\
\hline$\left\{C_{5}, C r\left(K_{3}\right), C r\left(P_{3}\right)\right\}$-free & 1 \\
$C r\left(K_{1,3}\right)$-free, not $\left\{C_{5}, C r\left(K_{3}\right), C r\left(P_{3}\right)\right\}$-free & $4 / 3$ \\
$C r\left(K_{1,4}\right)$-free, but $C r\left(K_{1,3}\right) \sqsubseteq G$ & $3 / 2$ \\
$C r\left(K_{1,5}\right)$-free, but $C r\left(K_{1,4}\right) \sqsubseteq G$ & $8 / 5$ \\
$C r\left(K_{1,6}\right)$-free, but $C r\left(K_{1,5}\right) \sqsubseteq G$ & $5 / 3$ \\
$C r\left(K_{1,7}\right)$-free, but $C r\left(K_{1,6}\right) \sqsubseteq G$ & $12 / 7$ \\
$\vdots$ & $\vdots$ \\
$C r\left(K_{1, r}\right)$-free, but $C r\left(K_{1, r-1}\right) \sqsubseteq G$ & $2-2 / r$ \\
\hline
\end{tabular}

Table 1: The values of $\max _{H \sqsubseteq G} \gamma_{p}(H) / \Gamma_{t}(H)$ provided by Theorem 5. The table is read as follows: If $G$ is a $\left\{C_{5}, C r\left(K_{3}\right), C r\left(P_{3}\right)\right\}$-free graph, the ratio equals 1. Otherwise, if $G$ is $C r\left(K_{1, r}\right)$-free, but not $C r\left(K_{1, r+1}\right)$-free, the ratio equals $2-2 / r$.

Corollary 2. Let $G$ be a $C r\left(K_{1, r}\right)$-free graph for some $r \geq 3$. Then

$$
\frac{\gamma_{p}(G)}{\Gamma_{t}(G)} \leq 2-\frac{2}{r} .
$$

This bound is sharp for each $r \geq 3$.

In particular, we obtain

Corollary 3. Let $G$ be a connected graph with maximum degree $\Delta \geq 2$ that is not isomorphic to $C_{5}$. Then

$$
\frac{\gamma_{p}(G)}{\Gamma_{t}(G)} \leq 2-\frac{2}{\Delta} .
$$

This bound is sharp for each $\Delta \geq 2$.

\section{The proofs}

\subsection{Auxiliary results}

The results of this paper are based on the following theorems.

In [17], the author gave the following characterization of graphs that hereditarily have an induced paired dominating set:

Theorem 6 (S. [17]). Let $G$ be a graph. Any induced subgraph $H$ of $G$ has an induced paired dominating set iff $G$ does not contain $C_{5}, \mathrm{Cr}\left(K_{3}\right)$ or $\mathrm{Cr}\left(P_{3}\right)$ as induced subgraph.

Furthermore, he gave a sufficient condition for a graph to have a total dominating set that induces a generalized claw-free graph: 
Theorem 7 (S. [17]). Let $G$ be a graph. If $G$ is $C r\left(K_{1, r}\right)$-free for some $r \geq 3$, then $G$ has a total dominating set $T$ such that $G[T]$ is $K_{1, r}$-free.

Let $G$ be a graph and let $M$ be a matching of $G$. If $M$ has maximal size among the matchings of $G$, it is said to be maximum. An augmenting path of $G$ is a path that starts and ends on unmatched vertices and alternates between edges of $M$ and $E(G) \backslash M$. A classical theorem by Berge [18] states a characterization of maximum matchings in terms of augmenting paths:

Theorem 8 (Berge [18]). Let $G$ be a graph. A matching $M$ of $G$ is maximum iff there is no augmenting path with respect to $M$.

In [19], Plummer and Saito prove the following bound on the matching number $\nu$ of a graph, defined as the maximal size of a matching:

Theorem 9 (Plummer and Saito [19]). If $G$ is a $k$-connected $K_{1, r}$-free graph, then

$$
\nu(G) \geq \min \left\{\frac{k}{r+k-1}|V(G)|,\left\lfloor\frac{1}{2}|V(G)|\right\rfloor\right\} .
$$

We need a special case of Theorem 9:

Corollary 4. If $G$ is a $K_{1, r}$-free graph for some $r \geq 3$, then

$$
\frac{\nu(G)}{|V(G)|} \geq \frac{1}{r} .
$$

Proof. Let $G$ be a $K_{1, r}$-free graph for some $r \geq 3$. We can assume that $G$ is connected. Theorem 9 gives

$$
\nu(G) \geq \min \left\{\frac{1}{r}|V(G)|,\left\lfloor\frac{1}{2}|V(G)|\right\rfloor\right\} .
$$

Since $r \geq 3$ and $|V(G)| \geq 2$ (by the general assumption that $G$ does not have isolated vertices),

$$
\frac{1}{r}|V(G)| \leq\left\lfloor\frac{1}{2}|V(G)|\right\rfloor .
$$

Thus

$$
\nu(G) \geq \frac{1}{r}|V(G)|,
$$

and this completes the proof.

We obtain the following observation:

Lemma 1. Let $G$ be a graph with a total dominating set $T$ such that $G[T]$ is $K_{1, r}$-free for some $r \geq 3$. Then there is a paired dominating set $P$ with

$$
\frac{|P|}{|T|} \leq 2-\frac{2}{r} .
$$

Proof. Let $G$ be a graph that has a total dominating set $T$ such that $G[T]$ is a $K_{1, r}$-free graph. We can assume that $T$ is minimal. Let $M$ be a matching of size $\nu(G[T])$ of $G[T]$. Since $G[T]$ is $K_{1, r}$-free, Corollary 4 gives

$$
\frac{|M|}{|T|} \geq \frac{1}{r} \text {. }
$$


Let $U \subseteq T$ be the set of unmatched vertices and let $u \in U$ be arbitrary. Then $G[T \backslash\{u\}]$ does not have isolated vertices, since otherwise $u$ would necessarily be matched to one of these isolated vertices. Since $T$ is a minimal total dominating set of $G$, there is a vertex $u^{\prime} \in V \backslash T$ whose only neighbor in $T$ is $u$. Otherwise $T \backslash\{u\}$ would be a total dominating set of $G$, too. We call $u^{\prime}$ a private neighbor of $u$. Let $P$ be the set obtained from $T$ by adding exactly one private neighbor $u^{\prime}$ for each $u \in U$. In $G[P]$, each $u \in U$ can then be matched to its former private neighbor $u^{\prime}$. Hence, $G[P]$ has a perfect matching and is therefore a paired dominating set of $G$. We observe that $M$ leaves exactly $|T|-2|M|$ vertices of $T$ unmatched. That is, $|U|=|T|-2|M|$. Thus, $|P|=|T|+|U|=2|T|-2|M|$. Together with (15) we obtain

$$
\frac{|P|}{|T|}=\frac{2|T|-2|M|}{|T|}=2-\frac{2|M|}{|T|} \leq 2-\frac{2}{r} .
$$

This completes the proof.

\subsection{Theorem 2, Corollary 1 and Theorem 3}

We need another auxilliary result first:

Lemma 2. Let $G$ be a graph and let $r \geq 3$ such that any minimal total dominating set of $G$ induces a $K_{1, r}$-free graph. Then

$$
\frac{\Gamma_{p}(G)}{\Gamma_{t}(G)} \leq 2-\frac{2}{r} .
$$

Proof. Let $G$ be a graph and let $r \geq 3$ such that any minimal total dominating set of $G$ induces a $K_{1, r}$-free graph. Let $P$ be a minimal paired dominating set of $G$ and let $M$ be a perfect matching of $G[P]$. Since any paired dominating set is a total dominating set, too, there is a minimal total dominating set $T \subseteq P$. Let $M^{\prime}=M \cap E(G[T])$ be the restriction of $M$ to $G[T]$ and let $U \subseteq T$ be the vertices of $T$ that are not matched by $M^{\prime}$.

Assume for contradiction that $M^{\prime}$ is not a maximum matching of $G[T]$. By Theorem 8, there is an augmenting path in $G[T]$ with respect to $M^{\prime}$. Hence, there is a bigger matching of $G[T]$, say $M^{\prime \prime}$, such that the set of unmatched vertices of $M^{\prime \prime}$, denoted by $U^{\prime}$, is a subset of $U$. Let $P^{\prime}$ be the set obtained from $T$ by adding the matching partner in $M$ of each $u \in U^{\prime}$. Clearly $P^{\prime}$ is a proper subset of $P . P^{\prime}$ is a dominating set, since $T \subseteq P^{\prime}$. Furthermore, $G\left[P^{\prime}\right]$ has a perfect matching and is therefore a paired dominating set. This is a contradicition to the minimality of $P$.

Hence, $M^{\prime}$ is a maximum matching of $G[T]$. As any minimal total dominating set of $G$ induces a $K_{1, r}$-free graph, $G[T]$ is also $K_{1, r}$-free. By Corollary 4, $\left|M^{\prime}\right| \geq|T| / r$. As clearly $|P|=|T|+|U|$ and $|U|=|T|-2\left|M^{\prime}\right|$, an easy calculation shows $|P| \leq(2-2 / r)|T|$. As $P$ was arbitrary, this completes the proof.

Theorem 2 is a simple consequence of Lemma 1 and Lemma 2:

Proof of Theorem 2. Let $G$ be a $K_{1, r}$-free graph for some $r \geq 3$.

By Lemma 2, (3) holds. 
We still have to prove (2). For every total dominating set $T, G[T]$ is also $K_{1, r}$-free. Hence, if $T$ is a total dominating set of size $\gamma_{t}(G)$, Lemma 1 provides a paired dominating set $P$ of size at most $(2-2 / r) \gamma_{t}(G)$. Thus,

$$
\frac{\gamma_{p}(G)}{\gamma_{t}(G)} \leq \frac{|P|}{\gamma_{t}(G)} \leq 2-\frac{2}{r}
$$

To see that (2) is sharp for each $r$, we observe that $T_{r-1}$ is $K_{1, r}$-free, $\gamma_{p}\left(T_{r-1}\right)=2(r-1)$ and $\gamma_{t}\left(T_{r-1}\right)=r$. An easy computation leads to

$$
\frac{\gamma_{p}\left(T_{r-1}\right)}{\gamma_{t}\left(T_{r-1}\right)}=2-\frac{2}{r} .
$$

Proof of Corollary 1. Since the proof of (4) is straightforward from Theorem 2, we proceed to the sharpness of (4). The case $\Delta=1$ is clear. For $\Delta \geq 2$, note that $T_{\Delta}$ attains the bound. Finally, (16) completes the proof.

For the proof of Theorem 3, we need the following Lemma:

Lemma 3. Let $G$ be a $\left\{C_{5}, T_{r}\right\}$-free graph for some $r \geq 3$. Then the subgraph induced by any minimal total dominating set of $G$ is $K_{1, r}$-free.

Proof. Let $G$ be a $\left\{C_{5}, T_{r}\right\}$-free graph for some $r \geq 3$ and let $T$ be a minimal total dominating set of $G$. Assume for contradiction that $G[T]$ is not $K_{1, r}$-free. That is, there is a subset $S \subseteq T$ with $G[S] \cong K_{1, r}$. Let $x$ be the dominating vertex of the $K_{1, r}$ and let $s_{1}, s_{2}, \ldots, s_{r}$ be the pendant vertices of the $K_{1, r}$. Since $T$ is a minimal total dominating set, each vertex $s_{i} \in S \backslash\{x\}$ has a neighbor $v_{i} \notin S$ such that $N\left(v_{i}\right) \cap S=\left\{s_{i}\right\}$. If there are some $1 \leq i<j \leq r$ such that $v_{i}$ is adjacent to $v_{j}$, then $G\left[x, s_{i}, v_{i}, s_{j}, v_{j}\right] \cong C_{5}$, a contradiction to the choice of $G$. Hence, $\left\{v_{i}: 1 \leq i \leq r\right\}$ is a stable set. Thus, $G\left[S \cup\left\{v_{i}: 1 \leq i \leq r\right\}\right] \cong T_{r}$, a contradiction to the choice of $G$.

We are now in the position to prove Theorem 3:

Proof of Theorem 3. Let $G$ be a $\left\{C_{5}, T_{r}\right\}$-free graph for some $r \geq 3$.

By Lemma 2, (7) holds.

We still have to prove (6). Lemma 3 shows that for every minimal total dominating set $T, G[T]$ is $K_{1, r}$-free. The rest of the proof is analog to the proof of Theorem 2.

Sharpness is attained by $\operatorname{Cr}\left(K_{1, r-1}\right)$ : We observe that $\gamma_{p}\left(C r\left(K_{1, r-1}\right)\right)=$ $\Gamma_{p}\left(C r\left(K_{1, r-1}\right)\right)=2 r-2$ while $\gamma_{t}\left(C r\left(K_{1, r-1}\right)\right)=\Gamma_{t}\left(C r\left(K_{1, r-1}\right)\right)=r$. This completes the proof.

\subsection{Theorem 4}

We need the following observation.

Lemma 4. For any graph $G$,

$$
\frac{\gamma_{p}(C r(G))}{\Gamma_{t}(C r(G))}=2-2 \frac{\nu(G)}{|V(G)|} .
$$


In particular, for any $r$,

$$
\frac{\gamma_{p}\left(C r\left(K_{1, r}\right)\right)}{\Gamma_{t}\left(C r\left(K_{1, r}\right)\right)}=2-\frac{2}{r+1} .
$$

Proof. Let $G$ be a graph. It is observed in [1], in the section dealing with paired dominating sets, that $\gamma_{p}(C r(G))=2|V(G)|-2 \nu(G) . \Gamma_{t}(C r(G))=|V(G)|$ is a straightforward consequence of the definition of corona graphs. These two facts lead to (17). The validity of (18) follows from $\nu\left(K_{1, r}\right)=1,\left|V\left(K_{1, r}\right)\right|=r+1$ and (17).

Further, we observe that

$$
\frac{\gamma_{p}\left(C_{5}\right)}{\Gamma_{t}\left(C_{5}\right)}=\frac{\gamma_{p}\left(C r\left(K_{3}\right)\right)}{\Gamma_{t}\left(C r\left(K_{3}\right)\right)}=\frac{\gamma_{p}\left(C r\left(P_{3}\right)\right)}{\Gamma_{t}\left(C r\left(P_{3}\right)\right)}=\frac{4}{3} .
$$

Proof of Theorem 4. By Theorem 6, the conditions 1 and 3 are equivalent.

Let $G$ be a graph. To see that condition 1 implies condition 2, assume that any induced subgraph $H$ of $G$ has an induced paired dominating set. Hence, $H$ has a paired dominating set which is a minimal total dominating set. Thus $\gamma_{p}(H) \leq \Gamma_{t}(H)$.

By (19), any graph that contains $C_{5}, C r\left(K_{3}\right)$ or $C r\left(P_{3}\right)$ as induced subgraph does not meet condition 2. Hence, condition 2 implies condition 3 and this completes the proof.

\subsection{Theorem 5, Corollary 2 and Corollary 3}

Combining Lemma 1, Theorem 4 and Theorem 7, we obtain Theorem 5:

Proof of Theorem 5. Let $G$ be a graph and let $\lambda$ be defined as in (8).

First we assume that $\lambda=2$, i.e. $G$ is $C r\left(P_{3}\right)$-free.

Further assume that $G$ is $\left\{C_{5}, C r\left(K_{3}\right)\right\}$-free. By Theorem $4, \gamma_{p}(H) \leq \Gamma_{t}(H)$ holds for any induced subgraph $H$ of $G$. Hence, (9) holds in this case.

We now assume that $G$ is not $\left\{C_{5}, C r\left(K_{3}\right)\right\}$-free. By (19),

$$
\max _{H \sqsubseteq G} \frac{\gamma_{p}(H)}{\Gamma_{t}(H)} \geq \frac{4}{3} .
$$

Since $\lambda=2, G$ is $C r\left(K_{1,2}\right)$-free by definition of $\lambda$. Now let $H$ be any induced subgraph of $G$. In particular, $H$ is $C r\left(K_{1,3}\right)$-free. Hence by Theorem $7, H$ has a minimal total dominating set $T$ such that $G[T]$ is $K_{1,3}$-free. By Lemma $1, H$ has a paired dominating set $P$ with $|P| /|T| \leq 4 / 3$. Hence,

$$
\frac{\gamma_{p}(H)}{\Gamma_{t}(H)} \leq \frac{|P|}{|T|} \leq \frac{4}{3}
$$

Combining (20) and (21) leads to

$$
\max _{H \sqsubseteq G} \frac{\gamma_{p}(H)}{\Gamma_{t}(H)}=\frac{4}{3},
$$

which is the desired equality (9) for the case $\lambda=2$. 
We now assume that $\lambda \geq 3$. Then, $G$ is not $C r\left(K_{1,2}\right)$-free and so (19) gives $\max _{H \sqsubseteq G} \gamma_{p}(H) / \Gamma_{t}(H) \geq 4 / 3$. To complete the proof, we have to show that

$$
\max _{H \sqsubseteq G} \frac{\gamma_{p}(H)}{\Gamma_{t}(H)}=2-\frac{2}{\lambda} .
$$

By definition of $\lambda, G$ is $\operatorname{Cr}\left(K_{1, \lambda}\right)$-free. Let $H$ be any induced subgraph of $G$. Then $H$ is also $C r\left(K_{1, \lambda}\right)$-free. By Theorem $7, H$ has a minimal total dominating set $T$ such that $G[T]$ is $K_{1, \lambda}$-free. By Lemma $1, H$ has a paired dominating set $P$ with $|P| /|T| \leq 2-2 / \lambda$. Therefore, $\gamma_{p}(H) / \Gamma_{t}(H) \leq 2-2 / \lambda$ and thus

$$
\max _{H \sqsubseteq G} \frac{\gamma_{p}(H)}{\Gamma_{t}(H)} \leq 2-\frac{2}{\lambda} .
$$

On the other hand, $G$ contains $C r\left(K_{1, \lambda-1}\right)$ as induced subgraph. Lemma 4 gives $\gamma_{p}\left(C r\left(K_{1, \lambda-1}\right)\right) / \Gamma_{t}\left(C r\left(K_{1, \lambda-1}\right)\right)=2-\frac{2}{\lambda}$. Hence,

$$
\max _{H \sqsubseteq G} \frac{\gamma_{p}(H)}{\Gamma_{t}(H)} \geq 2-\frac{2}{\lambda} .
$$

Now, (23) and (24) give (22). This completes the proof.

Proof of Corollary 2. Theorem 5 gives (10). Sharpness is obtained by $\mathrm{Cr}\left(K_{1, r-1}\right)$, as Lemma 4 shows.

Proof of Corollary 3. Let $G$ be a connected graph of maximum degree $\Delta \geq 2$ that is not isomorphic to $C_{5}$. If $\Delta=2, G$ is a path of length at least 2 or a cycle that is not $C_{5}$ and hence (11) holds by Theorem 4 . Sharpness is obtained by $P_{3}$, since $\gamma_{p}\left(P_{3}\right)=\Gamma_{t}\left(P_{3}\right)=2$. If $\Delta \geq 3, G$ is $C r\left(K_{1, \Delta}\right)$-free. Corollary 2 then provides (11). Sharpness is obtained by $C r\left(K_{1, \Delta-1}\right)$, as Lemma 4 shows.

\section{References}

[1] T.W. Haynes, S.T. Hedetniemi, P.J. Slater, Fundamentals of Domination in Graphs, Marcel Dekker, Inc., New York, 1998.

[2] E.J. Cockayne, R.M. Dawes, S.T. Hedetniemi, Total domination in graphs, Networks 10 (1980), pp. 211-219.

[3] M.A. Henning, A survey of selected recent results on total domination in graphs, Discrete Mathematics 309 (2009), pp. 32-63.

[4] T.W. Haynes, P.J. Slater, Paired-domination in graphs, Networks 32 (1998), pp. 199-206.

[5] J.A. Telle, Complexity of domination-type problems in graphs, Nordic Journal of Computing 1 (1994), pp. 157-171.

[6] T.W. Haynes, L.M. Lawson, D.S. Studer: Induced-Paired Domination in Graphs, Ars Combinatoria 57 (2000), pp. 111-128.

[7] B. Zelinka, Induced-paired domatic numbers of graphs, Mathematica Bohemica 127 (2002), pp. 591-596. 
[8] O. Favaron, M.A. Henning, Total domination in claw-free graphs with minimum degree 2, Discrete Mathematics 308 (2008), pp. 3213-3219.

[9] O. Favaron, M.A. Henning, Bounds on total domination in claw-free cubic graphs, Discrete Mathematics 308 (2008), pp. 3491-3507.

[10] O. Favaron, M.A. Henning, Upper total domination in claw-free graphs, Journal of Graph Theory 44 (2003), pp. 148-158.

[11] M.A. Henning, J. Southey, On a conjecture on total domination in claw-free cubic graphs, Discrete Mathematics 310 (2010), pp. 2984-2999.

[12] P. Dorbec, S. Gravier, M.A. Henning, Paired-domination in generalized claw-free graphs, Journal of Combinatorial Optimization 14 (2007), pp. $1-7$.

[13] P. Dorbec, S. Gravier, Paired-domination in subdivided star-free graphs, Graphs and Combinatorics 26 (2010), pp. 43-49.

[14] M. Blidia, M. Chellali, O. Favaron, Ratios of Some Domination Parameters in Graphs and Claw-free Graphs, pp. 61-72, In: A. Bondy, J. Fonlupt, J.L. Fouquet, J.-C. Fournier and J.L. Ramírez Alfonsín (Eds.), Trends in Mathematics: Graph Theory in Paris, Birkhäuser, Basel, 2007.

[15] R.C. Brigham, R.D. Dutton, Domination in claw-free graphs, Congressus Numerantium 132 (1998), pp. 69-75.

[16] P. Dorbec, M.A. Henning, J. McCoy, Upper total domination versus upper paired-domination, Quaestiones Mathematicae 30 (2007), pp. 1-12.

[17] O. Schaudt, The structure of total dominating subgraphs, submitted manuscript (2010).

[18] C. Berge, Two theorems in graph theory, Proceedings of the National Academy of Sciences of the United States of America 43 (1957), pp. 842 844.

[19] M.D. Plummer, A. Saito, Forbidden Subgraphs and Bounds on the Size of a Maximum Matching, Journal of Graph Theory 50 (2005), pp. 1-12. 